\title{
A Spatial-temporal Epistemology of Four-dimension Transportation Issue
}

\author{
Moreno Ferrarese
}

Department of Economics,University of Verona , Italy

Copyright $@ 2015$ Horizon Research Publishing All rights reserved.

\begin{abstract}
The article illustrates the answers to the questions on the transportation genesis, through the definition of a multi-dimensional space and of time. Practically, it reveals and explains the forth dimensional parameter - psicometric. And to introduce this finding, an authentic epistemologic approach is applied. The new method is raised through a scientific journey across the knowledge theory - epistemologic time assumptions in the philosophic, mathematic and physical frameworks to reach the conclusion of the perceived time formalization. The author proposes a speculative and refined analysis, far from over, but just in the beginning, in a confused scenario of a subject, as the logistics and transport economics, which is evolving time by time over the size of the ruling scientific opinion.
\end{abstract}

Keywords Epistemological Theory, Multi-Dimensional Space and Time, Transportation Science

\section{Introduction}

To speak of transportation has become a conspiracy, always in fashion: they all deal with transportation, everyone is talking about transportation boasting its competence. Transportation is now on everyone's lips.

During the conferences they listen to the "subject matter experts" who present their analysis and offer many innovative solutions to old problem that has gripped our planet: pollution from transport and traffic congestion in cities.

Of course, these problems are important because they touch the quality of life of citizens and the income of families and businesses. But the question always arises: why you carry? And, above all, what's the transport? The word derives from the Latin trans-portare, which means "through", and "carry". The transport is, in general, to offset an imbalance that is created naturally or deliberately generated between territories (surplus/deficit area) so that, with the transport term, it is pointed out, often, the movement of people and goods from one place to another. Not a random movement, messy, but motivated. Often driven by habits and "adjusted" from inter-legal conditions. According to ISTAT ${ }^{1}$ classification, distinguishing the movement motivated to "work", "business", "study", "tourism", "commission", transport and mobility are the transfers over time and through space of people and of things from "the residential development" to "the production facilities", for "the community facilities" and between them.

\section{Some Reflections on Space and Time}

So far we have discussed the transport properties, but not its nature. We still have not answered the question "What is transportation"?

There are two components, the complex itself, which define the transportation as the physicists, the mathematicians and the philosophers know well: the space and the time, plus a third component which is the physical object of the transfer, without which ${ }^{2}$ the event itself ${ }^{3}$ won't produce. It should ${ }^{4}$ be, in particular, a path from one point to another in a space bound planar $^{5}$, to perform a displacement material and it must take some time.

Space, by definition, is given by the Euclidean ${ }^{6}$ surface,

${ }^{1}$ http://www.istat.it/it/archivio/spostamenti.

2 Necessary condition (formally, a proposition $\mathrm{P}$ is a necessary condition for $\mathrm{Q}$ if $\mathrm{P}$ implies Q).

${ }^{3}$ The man has an integral perception of space and a fully differential perception of time. [Author's note: for us, outside observer] When looking at a distant object, the eye catches in a single image, both that is near the object and that is along the line of sight, and between the eye and the object behind it (the so-called "depth of field"). If, however, we consider a temporal event, it will never have a complete perception. The experience teaches, in fact, that time is perceived only through the sequences of moments. Recalling an event, you do not remember ever to what has happened between then and the present moment. In other words, there is a perception (and memory) instantaneous or "differential" of time (Giuseppe Longo, Some Aspects of Time in Physics and Philosophy, or some thoughts of a physicist on a problem as old as time, paper, 2008).

${ }^{4}$ Sufficient condition (formally, a proposition $\mathrm{Q}$ is sufficient for $\mathrm{P}$ if $\mathrm{P}$ implies Q).

5 With only two Cartesian coordinates, while the third never enters into the representation of static or kinetic transport logistics. To identify a point on a plane you must specify its two Cartesian coordinates (abscissa and ordinate), to identify a point in space it takes three (abscissa, ordinate and height), but to specify a physical event you must specify four numbers. The world of events, then, is not the abstract geometry of solids, in which three dimensions would be sufficient, but a world of four dimensions: three of space and a fourth of time (Giuseppe Longo, ibid.)

6 Euclid's Elements $(\Sigma \tau$ oเ $\chi \tilde{\varepsilon} \alpha)$ Book I, Definitions. In the space of 
"an area [Author's note: that] is what has only length and width." For mathematical reductionism ${ }^{7}$, it is distinguished by a surface portion, as the line, defined as "a length without width" 8 .

The transfer "material", then, is expressed as the linear displacement-distance ${ }^{9}$ in time ${ }^{10}$.

But what time ${ }^{11}$ ?

The transformation of society - it is thought of the transition from working in the fields to the artisan and merchant, to city development, to the economic and cultural transformation - sets different values than in the past and stimulates the new ideas to the contemporaries.

Euclidean geometry, the fundamental invariant is the distance between two points. $P^{(1)}=\left(x_{1}^{(1)}, x_{2}^{(1)}, x_{3}^{(1)}\right)$ and $P^{(2)}=\left(x_{1}^{(2)}, x_{2}^{(2)}, x_{3}^{(2)}\right)$

Indeed, the usual Euclidean space is defined starting from the invariant of the Euclidean distance, whose square is:

$\Delta S^{2}:=\Delta x^{2}+\Delta y^{2}+\Delta z^{2}=\sum_{i=1}^{3} \Delta x_{i}^{2}$ con $\Delta x i:=x_{i}^{(2)}-x_{i}^{(1)}$

In these cases, this quantity does not change when applied to the translations:

$x_{i}^{\prime}=a_{i}+x_{i} \quad i=1,2,3$

In Euclidean geometry and physical pre-relativistic the length of an object does not change when it moves or rotates in space.

7 According to the axiom of Zermelo-Fraenkel: given a generic set A, there exists a set $\mathrm{B}$ such that, given a generic set $\mathrm{C}, \mathrm{C}$ is an element of $\mathrm{B}$ if and only if $\mathrm{C}$ is an element of $\mathrm{A}$ and $\mathrm{P}$ is true for $\mathrm{C}$. Let $\mathrm{P}$ be a predicate in a generic variable that does not use the symbol $\mathrm{B}$. Then, in the formal language of the Zermelo-Fraenkel axioms, the axiom reads:

$\forall A, \exists B, \forall C: C \in B \Leftrightarrow C \in A \wedge P(C)$

(A. Fraenkel, Y. Bar-Hille, A. Levy, Foundations of Set Theory. Fraenkel's final word on ZF and ZFC, North Holland, 1973 ).

8 Euclid's Elements $(\Sigma \tau$ o $\chi \chi \varepsilon \tilde{\tau} \alpha)$ Book I, Definitions.

9 The distance normally considered in $\mathbb{R}^{2}$ is the Euclidean one, equal to the square root of the square of the difference horizontal (between the two points) plus the square of the vertical difference: $d=\sqrt{\left(x_{B}-x_{A}\right)^{2}+\left(y_{B}-y_{A}\right)^{2}}$

If you delete the second dimension this function is reduced to the form of the difference between two numbers: $d(x 1, x 2)=|x 1-x 2|$ also called Manhattan distance, which generates the so-called geometry of the taxi.

10 "What, then, is time? If no one asks me, I know. If I had to explain to anyone who asks, I do not know "(St. Augustine, Confessions).

11 The ancient Greeks had two words for time: kronos and kairos. While the first refers to the logical time sequence, the second means "a time in between," a moment of undetermined period of time in which "something" special happens. Namely, the special thing depends on who uses the $\lambda \dot{\varepsilon} \gamma \varepsilon i v$ (léghein). Who uses the $\lambda \dot{\varepsilon} \gamma \varepsilon \dot{ }$ v defines it, the being of the thing. Who defines the special thing defines being of something special. There are, therefore, precisely the same words, which define being as special.

While the kronos is quantitative, kairos has a qualitative nature. With St Augustine, time becomes a psychological individual fact. Through memory we can distinguish between the past that is no longer and present, and with the expectation we can get an idea of the future that is not yet.

For Kant, however, time is always in need of a symbolization through the space. He shows that we need space to take over and learn about our existence. The spatial representation of time is, therefore, a need considered as absolute, without which the time could not be known. And, given that we are obliged to reveal the time to draw a mental line, the traction of the body must necessarily intervene, but always an internal mobility, lived from within, and which we can call "transcendental". Thus Kant distinguishes a movement in space and motion generator of space, such as the movement determining an object and the movement as an act of the subject (Transcendental Analytic, 24). The space is the necessary condition of our stay and our stability. In the pre-critical period Kant had left a Leibnizian conception of space that was understood as the relationship between places, namely, between the positions of two objects: the space was something relative, being defined non by the term of the reference absolute and unitary, but simply by the distances and mutual relations of things.
During the fourteenth century, with the diffusion of the mechanical clock, the differentiation of a "secular time" from the "church time" then in effect (i.e. the time of Gloria, the Hail Mary or a Requiem ) came: the secular time, namely that regulates life and human activities, requires a measurement unit different from that which is connected with the natural or religious events ${ }^{12}$. Thus, it is required an iterative constant rhythm, synchronized and often synchronous between events, but surely the same for all, that is a time dimension that involves all individuals at the same time, possibly with the same events.

So (and here in Europe) it is born the mechanical clock ${ }^{13}$, which, compared to the oldest time-marking instruments, defines a constant flow of time, equator, without reference to sunlight, and which, according to the seasons, makes unequal the length in the Tropics ${ }^{14}$. The time thus becomes diatopic and diachronic, as a concept detached, "abstràctus", released by the concrete events.

Although human activities are no longer related to the natural and social phenomena, unspecified in their lifetime, but, rather, to the clock running which can provide them a well-defined timing.

Thus, the time quantity, for which in the past, even in the most recent one, it was necessary to perform a particular activity, was decreased significantly and includes within it the potential to be decreased more and more, thanks to the new components of the integrated communication and innovative tools, as well as through the timely adoption of Zeitsparendetrick ${ }^{15}$ tricks-save-time, as the synchronization of events. The result which we find is that of a world zipped, a world, i.e., in which the distances between the persons and the places apparently (further we shall understand why) are compressed.

We think, also, about any system or means of transport and, by comparing the travel time today with the necessary time 70 years ago, we realize that no era, as the present one, is capable to generate time ${ }^{16 .}$

But a more careful anthropological study will lead us to

12 The civilizations of the past, such as the Egyptian, the Babylonian and especially the Aztecs, had no sense of time. These civilizations were able to distinguish the succession of days, years and seasons. In measuring them, they attributed to the rhythms of daily life, social or public the intervention of the gods that men hoped to ingratiate himself with prayers, sacrificial rites and initiations also human.

13 The mechanical clock seems to have been born between 1230 and 1270 , according to the most ancient treatise on English, the clock is from 1271.

14 While in the land polarity, following the rotation of the Earth about 24 hours and a precession motion very slow - its axis of rotation rotates with a cycle of 25,800 years around the perpendicular to the plane of its orbit, with respect to which is inclined for approximately $23^{\circ} 27^{\prime}$, with nutations of 18.6 years - the perception of passing astronomical time is less perceived.

${ }^{15}$ The hypothetical imperative of industrialized societies is synchronization, which means a temporal coordination of multiple levels: international level, thanks to time zones that divide the earth into 24 zones of 15 degrees each and differentiated exactly one hour one from 'other and to GMT hour; the national and local level, through synchronization of mobility management (of transport urban and non-urban, of working hours, of the provision of services, etc.). The synchronization is the engine for operation of a complex social system, a society that was equipped with a single collective horizon, with a unique messianic vision, socially objectified and subjectively real.

${ }^{16}$ Peri P., Progetto di ricerca su esigenze e aspettative degli utenti giornalieri delle ferrovie rispetto ai servizi loro offerti e ai materiali rotabili utilizzati, Dipartimento di Sociologia e Ricerca Sociale dell'Universita' degli Studi di Trento, 2001. Transcendental Analytic, 24). 
think that the companies producing goods and services without the solution of continuity (especially those aimed at saving time) make the experience of the lack of time, much more than an archaic company where the rate of productivity or efficiency is lower. Nothing is truer. Therefore, the harm, declined from a syntactic self-referential construct, seems to cause itself: the more you produce, the more you work, the more time you consume ${ }^{17}$.

Even if the current form of non-embryonic human biological life is aimed at the survival exasperated of the species throughout its natural evolution, would lead us to live "time consuming" without having to think about the real human condition and the awareness of impending death, just like it does for infants and children, or for the contemplative cultures, just aware of the discovery of human frailty, which is acquired through psychological growth typical of childhood. It leads us, to tell it Toreau ${ }^{18}$ to "wipe out everything that wasn't life and not to discover the point of death, that I had not been living .. ".

Here, then, that the categorical imperative ${ }^{19}$, the eternal condemnation of Western culture assails us in there to live with the arrogance of the event that reminds us, to live for the tyranny of time ${ }^{20}$ that it is determined. The living is no longer marked by Kaıpó ${ }^{21,}$ but Kpóvo $\varsigma^{22}$. It calls us back to the categorical imperatives of post-modern society: in that company the technology replaces the capital, production and proliferation of signs symbolic and of the images simulated replaces the production of goods ${ }^{23}$.

In the post-modern society the social subject appropriates the property to boost its personality and to create so an image fictitious and false of itself, in order to show off, that would undermine social relationships ${ }^{24}$, causing an implosion of society itself.

Consequences of what appears reification, Versachlichung of things, the alienation of individuals, the spread of

\footnotetext{
${ }^{17}$ Paolucci G., Una figura della temporalità moderna: la scarsità del tempo, in Belloni M.C., Rampazi M. (a cura di) Tempo, spazio, attore sociale. Tredici saggi per discutere. Franco Angeli, Milano 1989, pag. 166.

18 "I went into the woods to live deliberately, to live deeply and suck all the marrow of life, to rout all that was not life and not find out, on his deathbed, that I had not lived" (HD Thoreau, A Week on the Concord and Merrimack Rivers / Walden / The Maine Woods, Ticknor and Fields, Boston, 1854).

19 A categorical imperative denotes an absolute, unconditional requirement that states its authority under any circumstances, necessary and justified as an end in itself. Authority given by the formula: "Act only according to the maximum, for which you can and at the same time you want, and which becomes an universal law." (I. Kant, trans. James W. Ellington [1785], Grounding for the Metaphysics of Morals, $3^{\text {rd }} \mathrm{ed}$.).

${ }^{20}$ Time does not exist, it is only one dimension of the soul. The past does not exist because there is no more, the future does not exist as yet to be and the present is just an instant non-existent of separation between past and future (St. Augustine, Confessions).

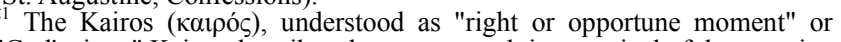
"God's time." Kairos describes the retro-causal time, typical of the negative solution of Klein-Gordon the whose advantage is to treat time and space in the same way, while d'Alembertian operator (as an operator of the waves, or the extension of the Laplace operator in the space of Minkowski) appears to be an invariant. According to Pythagoras Kairos is the basis of intuition and the ability to anticipate the future and to choose into advantage.

${ }_{22}$ Kronos describes the sequential time, familiar to us, typical of the positive solution of the Klein-Gordon, moments of absolute fact that flow from past to future. Supercausal Aion, at last, describes the time in which the past, present and future coexist (the time of quantum mechanics, the sub-atomic world).

${ }^{23}$ Baudrillard J., La società dei consumi, Il Mulino, Bologna, 1976.

${ }^{24}$ Ibidem.
}

conformity, of uniformity oral and gestural, as social and value approval that determines the end of transcendence, seen as the inability of the subject to perceive and to alert the authentic and real needs, and therefore bind to "God's time".

Life itself, therefore, articulated by the rhythm of the seconds, condemned the individual biographies and collective symbolic universes to a race "timeless", to an end unknown, insinuating, through the power of suggestion, the eternity behind every gesture. One lives time "with bated breath" for the illusion of being eternal, so it can suck the marrow of life itself ...

We constantly look for the speed, the relationship of the compression space/time, a kinetic of the event rather than its static version, in order to compress the vital time, stretching, thus, in the collective imagination to the individual participation and perceived life. And in the Western society, where to have is more important than to be 25 , time becomes a commodity economy and variable key that influences the production of goods and services, consumption of goods and services, financial intermediation, the accumulation of wealth, redistribution of income and wealth, insurance ${ }^{26}$.

Even the physical death of the individual doesn't interrupt his spatial and temporal relationship with reality: each new chemical cluster, formed by the lifeless body, becomes the temporal form of collective surviving memory, a new event, the same time. And you try to "cheat Death" by using the "life-saving tricks" ${ }^{27}$, forgetting time, "jumping over its own thinking" ${ }^{28}$, accelerating all human activities, supervising always a greater amount in the shortest possible time ... To travel in order to forget, to never have to $\mathrm{die}^{29}$.

So it is, the time-memory, the two-dimensional space and the object of transport are the complex components of a relationship of elemental composition defined by the physical as speed ${ }^{30}$. And the speed at which they are transported is the ratio of different units of measure in the nature and properties, including the distance traveled, expressed in kilometers ${ }^{31}$ or submultiples 32 and the time expressed in seconds or overmultiples. So, once again

${ }^{25}$ Erich Fromm, To have or to be? (trad it. Avere o essere?), Mondadori, Milano, 1980.

26 According to the classification and definitions of standard SNA, UN and ESA of the European Union (ISTAT, the Italian Accounts, Bologna, Il Mulino, 2001.

27 The well-known Russian time economy and tricks.

28 "This beautiful impediment, the thought of thoughts, if those who created it? The very life if one created, its chief impediment: now it jumps beyond its own thinking "(F. Nietzsche, Ditirambi di Dioniso, Adelphi, Milan, 1988, p. 133).

29 "Cast your burden in the deep! / Man! Forget! Man, forget! / Divine is the art of forgetting! / If you want to fly / if you want to be at home in the heights / jumps into the sea that which is heavier in you! / There is the sea, thrown into the sea! / Divine is the art of forgetting "(F. Nietzsche, ibid, p. 139).

30 The relationship between space and time is defined as speed, which, in transportation, assumes the aspects of "commercial speed" if we add also the time spent for vehicular entry and exit of persons or for handling of goods, or "door-to-door " speed if we include the time to access the transport network or system.

31 In the MKS system. This system of physical quantities and measurement units began in 1889 with the 1st CGPM: then called "MKS system" because it included only the fundamental units of length (meter), mass (kilogram) and time (seconds).

32 We refer only to European measures, non to Anglo-Saxon or other Eastern. 
talking about a relationship of "relativity" between "immutable" space and "immutant" time.

In fact, as we mentioned, the space and time, as perceived by non-disabled individuals, follow the law of Minkowski ${ }^{33}$ of space and time reduced to three dimensions ${ }^{34}$, corresponding to the description of events, with that ordinary Euclidean law. Everyone, well, perceives time "fleeing" ${ }^{35}$ as its carpe diem, as its mode to live the present with a relative time. So if the temporal regression happens with the memory of the event described kinetically, the future event, still

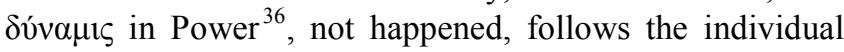
experiential screening collectively objectified.

To that it applies the restricted relativity ${ }^{37}$ of Grossman ${ }^{38,}$ according to which the event kinetically describable contracts minkowskian space-time upon increasing of the speed of transfer. How often we hear: "I arrived in no time"! Or, "I've used nothing".

Or, conversely, if the speed is low you hear the words: "It took me a lifetime get"!

Each culture also has its own representation of the speed of transport. Each time of the perception of an event is filtered by the led of values of the afferent culture: in Indian contemplative culture, for example, the delay of a train means computable only after twenty-four hours from the hour of arrival on the station.

33 "The concepts of space and time, which I want to expose, derive their origin in the soil of experimental physics, and therein lies their strength They are radicals. Henceforth, space intended individually and time intended individually are destined to fade into nothing but shadows, and only a connection of the two will preserve an independent reality" (Hermann Minkowski, speech of 21 September 1908 at Assembly of Nature Scientists and Germans Doctors). Even the concept of simultaneity loses its character of absoluteness, because if the speed of light is finite and it is the same for every observer, two simultaneous events in an inertial frame are no longer so when viewed from another inertial system, in motion than that. Likewise, the events become different if observed by the internal observer or by an outside one.

34 As in any model of time-space, each point in space has four coordinates $(\mathrm{x}, \mathrm{y}, \mathrm{z}, \mathrm{t})$, three of which represent a point in space, and the fourth - a precise moment in time: intuitively, each point represents an event, fact that happened at a particular place at a precise moment. The movement of an object point is then described by a curve, with increasing time coordinate.

35 "O virgin seize the fleeting moment / Take the rose when it's time / that time, you know, fly / and the same flower that blooms / today / tomorrow will wither" (Horace, Odes 1, 11, 8).

36 From Aristotle, perfect action, which carries its own end, this act is final or final realization (or entelechy). The movement is the process that takes time, what was $\delta u v \alpha \mu \iota \varsigma$ - potentially, the entelechy is the final deadline $(\tau \varepsilon \dot{\lambda} \lambda \mathrm{s})$ of the movement, its perfect completion. But, the entelechy as such, is also the realization accomplished, and then the perfect form of what becomes; it is the species and the substance together. The act is identified, therefore, with the form or species, and when this is a perfect act or final realization, it is identified with the substance. This is, then, the same current reality and the principle of it. In front of it, the matter considered in itself, i.e. as a material or raw material, virtually devoid of actuality or form, is not to

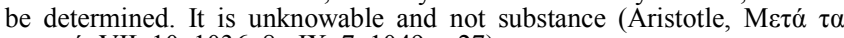

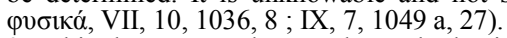

37 This theory turned out to be at the beginning of 900 , an expansion of classical mechanics, which is contained in the so called. special relativity and that can be tested only at speeds much lower than that of light. The loss of the concepts of absolute space and absolute time has resulted in the relativistic contraction of the lengths and dilation of time to nearly the photonics speed.

Moreover, while in classical mechanics space and time are treated as substantially separate entities, the relativity introduces the concept of space-time in which space and time are inextricably linked together (in classical mechanics it is possible to consider the time regardless of the space, and vice versa).

38 Marcel Grossmann (Budapest, April 9, 1878 - Zurich, September 7, 1936) was a Hungarian mathematician.

\section{An Analysis of Space Dedicated to Transportation}

After exploring the full space-time relationships of event, we begin to analyze the space (land, water and air) dedicated for transportation. We note how this is increasingly specialized and equipped for the spaces added to other human activities that generate income, culture and socialization. The summation of open spaces, transportation and service to the territory, determines a hyperspace ${ }^{39}$ within which the acceleration of the local economy with income, price, market share, system ${ }^{40}$ effects happens. To put it as the econometrics of transport, there is a direct correlation between independent endogenous and exogenous variables of the economy (without treating too any eventual and natural multicollinearity or the independence of independents, in the area of the assumptions of Ordinary Least Squares ${ }^{41}$ or TSLS $^{42}$ - in the worst case estimation, through the use of $\mathrm{GLS}^{43}$ - estimators corrected, with fitting

39 Although two points in space in 3 dimensions may appear very distant, the same points in a hyperspace, with a higher number of dimensions, are connected by a path length considerably shorter. That is, what looks initially like a geodetic in 3 dimensions, not a true geodetic hyperspace - the concept of geodetic is intimately related to the Riemannian metric - that is connected with the concept of distance and acceleration. In this way, also not passing the speed of light, it would be possible to travel the great distances in a reasonable time. A geodesic is the analogue of a straight line in the usual Euclidean space (or plane). It is a differentiable curve $\alpha$, which minimizes the length locally. More precisely, each $t$ inside the domain [a, b] has an internal $U$ such that the distance between $\alpha(t)$ and $\alpha\left(t^{\prime}\right)$ is equal to the length of the sub-arc $\alpha$ connecting the two points, for each $t^{\prime}$ in $U$ (Kobayashi S., K. Nomizu, Foundations of Differential Geometry, Vol. 1, Wiley-Interscience, 1996).

${ }^{40}$ The effects we observe, in the interaction between economics, land use and transport, are the following:

- on the price: when one transfers the transportation on pricing of goods and services of the additional costs that we find, then, on the final sale price;

- on the market share or locational effect: when entering into a new transportation system or infrastructure, its improvement produces the effects on diverted or generated traffic by attracting new mobility and demonstrating so that mobility, ejected by an arc of the network or from one mode of transport, pouring on other arcs of the network or transport modes, creates interdependence between regions and between individual halos in which these are divided;

- on the income: when transport injects a "value added of transport," it brings the significant improvements in the efficiency of the production system, shortening the time of placement of goods on the market with immediate effect on the provision and management of stocks;

- on the system: for the synergies that are to be active.

It is to consider also the effects of transport on the same business strategies.

The interactions between transport and their environment are, in general:

- the choice of firm size;

- the choice of companies technology, with obvious problems of polarization or decentralization of production activities;

- access to new markets and better penetration into existing ones;

- the level of investments and financial assets;

- the quantity and, especially, the quality of goods introduced on the market, considering the impact on the choices of production and storage of perishable goods.

As it is easily understood, the problem of transport can't, nor should be, neutral with respect to the choice of production, to the consumption patterns, to the structure of the long period of economic system, to the same regulatory framework. In several cases, the modifications introduced by the transport in the processes of production, in the organization of markets, in buying behavior of consumers and in international economic relations reveal the effects that are likely to become irreversible and, therefore, to rule in a broader, systemic context (Ferrarese M., Course of Economics of Transport and Mobility - CPS, TransmitWorld, Verona, 2010).

41 Stock J., Watson M., Introduzione all'econometria, Pearson Education, Milano, 2005, pp. 100.

42 Method of two-stage least squares

43 Generalized Least Squares of Aitken. Alexander Craig Aitken (Dunedin, 1 April 1895 - Edinburgh, November 3, 1967) was a statistician from New Zealand, 
close to unity ${ }^{44}$ ) and the dependent transport variable, next to the unit value. This means that the transport is $\tau \dot{\varepsilon} \lambda o \zeta$, that is necessary and sufficient condition [see: Notes 2 and 4] to obtain the economic effects of land (Entelechy), while a

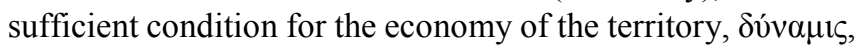
is to get transportation.

To obtain and deliver the economic effects of land it is necessary to create the fixed structures on which we shift the transport unit, with the load units (especially in the intermodal logistics platforms), with movement units 45 . They apply to the physical laws of dynamics of non-Newtonian fluids, whereas considering the transport vehicles as particles of the fluid and the transport infrastructure as pipelines: the equations of continuity ${ }^{46}$ or "mass balance", Bernoulli's theorem ${ }^{47}$, related to speed of the

$44 \mathrm{R}^{2}$ does not measure if indeed there is a relationship (any kind) between $y_{i}$ and the regressors, but only the extent to which a linear model can approximate the reality of the observed data. A nonlinear model, for example, could represent more effectively the relationship between the dependent variable and regressors, showing a good explanatory power, even in the presence of an $\mathrm{R}^{2}$ close to zero. It can be shown that adding regressors to the model can only increase the value taken by $\mathrm{R}^{2}$ :

This does not mean that the new model found is better than the previous one, meaning that these best factors compete to explain the dependent variable, but only that the best fitting was made in an artificial way. In order to correct the measure of goodness of the fitting (adaptation) indicated, so as to penalize a model characterized by a large number of regressors, certain statistical packages report a correct $\mathrm{R}^{2}$, defined as:

$$
\bar{R}^{2}=1-\frac{N-1}{N-k-1}\left(1-R^{2}\right)
$$

It is evident that, by increasing the number of regressors $k, \mathrm{R}^{2}$ in general decreases, correcting the artificial increase of $\mathrm{R}^{2}$. It could be also shown that $\mathrm{R}^{2}$ increases, adding a regressor, only if the value of t-statistic associated with the coefficient of that regressor is greater than 1 , so that the value of $\mathrm{R}^{2}$ is properly linked to the significance of additional variables.

45 (Ferrarese M., ibid, 9th lesson).

46 Example of mass balance application: the velocity to escape from the traffic congestion in urban areas increases if lateral encumbrance rises. Just after the mobile highway construction sites, provided that indicated, the escape velocity of the motor vehicles always increases by decreasing the safety distances in the omotahic queues in the vicinity of the jam, in agreement with the linear patterns of Greenshield or logarithmic of Greenberg or that of Underwood. In the vicinity of the restriction, the density (by experimentation) rises to a $\mathrm{Kb}$ value due to the reduced speed that allows lower clearances (condition of "tail" on the move, or supercritical flow), while just after the obstruction one has a normalized density $\mathrm{Km}$ lower to that in stationary conditions.

The propagation of the different states of flow (defined by a triplet of speed, density and flow) occurs according to waves which generally are considered (by approximation) punctual, and which are defined the kinematic waves. From the place of occurrence a so-called "shock wave" W start upstream, which will invest the vehicular current with normal density $\mathrm{K}$ and at any instant separates this current from the current in state of the queue. Since the number of vehicles in a time interval $t$ that are invested by the wave is constant, it follows that: $k_{a}\left(V_{a}-W\right) t=k_{b}\left(V_{b}-W\right) t$, then the speed of the shock wave is given by $W=\frac{Q_{a}-Q_{c}}{K_{a}-K_{b}}$, that is the slope of the chord joining the point on parable of the flow / the density just before of the event (A) with that, on the branch "congested" corresponding to the restriction (B).

Once the occurrence removed another wave with a speed greater than the previous starts which will reach the point of maximum capacity $(\mathrm{C})$. When this wave reaches the shock wave the queue will be dissipated and the last wave from $C$ to A will bring the system to initial conditions of flow (reduced from: Wikipedia, http://it.wikipedia.org/wiki/Deflusso_veicolare ).

$47 \mathrm{In}$ the case of land transport it is possible to neglect the term gravitational of Bernoulli equation because the lines of traffic flow to which reference is efflux torricellian ${ }^{48}$ according to the criterion of simplification given by Novacula Occami ${ }^{49}$.

The fixed structures mentioned are artificial artifacts (such as roads, highways, railways, pipelines, cable railways, canals) and natural sites (pools open or closed), or technical or legal agreements (air routes), classified by the means of transport that use them. So the road, or strata (Latin for "stratum"), is an infrastructure deputed to the road and highway transport, a way for means of transport to guide free, standardized on the Roman road. The railways are the ways for means of transport invented to guide constrainedly by carriage. The channels are free ways to guide by means of the dynamic flow transportation. The stretches of open (sea) or closed (lakes) water are infrastructure for means of transport to guide free by hydrodynamic transport. The air routes are the serial spatial positions, intercepted by means of transport to guide free in the variable currents dynamic conditions.

There are, moreover, the static structures of the means of shelter for maintenance and service operations (ports, airports and service) or of modal and system integration with defined intermodal logistics platforms or marine terminals for freight (on ground) and off-shore if in water. In such cases, the standardized load units (in the case of intermodal freight) or less (in the case of multimodality goods) through the units of displacement are integrated to the transport unit restarted. All this is defined as logistics ${ }^{50}$. In fact, the

made to have approximately the same potential energy.

48 The law of Torricelli says that the speed of a fluid in output from a hole (of very small section compared to the size of the container) is equal to the square root of the double product of the acceleration of gravity and of the distance " $h$ " between the free surface fluid and the center of the hole that has been practiced:

$$
v=\sqrt{2 g h}
$$

The speed is equal to that which the fluid would have during a free fall from the height " $h "$. The equation of Torricelli can be obtained by calculating the differential of this formula, less one arbitrary constant (which is the initial velocity vi). The only variable terms are the speed $v$ and height $h$. In road transport we replace the height $h$ with the length $L$ of the traffic section, the gravity (in the land transport), is ignored as irrelevant.

${ }^{49}$ Occam's razor: it imposes to avoid any additional assumptions when those initials are enough. If a theory works, it's useless (and harmful) to add a new hypothesis.

${ }_{50}$ The term logistics is derived from the greek term logos ( $\lambda$ ó $\left.\gamma \circ \varsigma\right)$ which means "word" or "order".

For the Greeks, the two concepts were closely correlated and expressed with the same term. Lógos also derives from "logic" i.e., the study of the arguments and the way in which they are correct. Currently, the term "logistics" means:

- The organization, planning, execution and control the flow of goods, and of the related information, from development and from procurement through production and distribution to the end customer, with the aim of meeting the demands of market at minimum cost and with less use of capital (definition of the European Logistics Association).

- Logistics is the set of organizational, managerial and strategic policy that govern the flow of farm equipment (and information) from the purchase of raw materials from suppliers through to delivery of finished products to customers and to the after- sales services (definition of the Italian Association of Logistics).

- Logistics is that part of the "supply chain" (supply chain) who plans, manages and controls in an efficient and effective way the flow of goods and services and related information from point of origin to point of consumption with the aim of meeting the customer requirements (definition of Council of Logistics-Management).

- Logistics is the discipline that has as its goal: "the use of the exact method for making available the exact amount of the exact material in the exact place at the right time, in the right sequence, in the right position, under the right conditions and right cost". 
logistics like, for themselves and for their discipline, to give other definitions.

\section{Conclusions}

In the academic environment it's defined as "Science" very often wrongly - what recourses only to the mathematical process, for methodological reductionism ${ }^{51}$ in the formal phenomenal description (ignoring the "Logic", that is the epistemological approach ${ }^{52}$ ): this could also appear to be true if the mathematician process were applied, for our argument, to the engineering and manufacturing of transport infrastructure and logistics platforms. In such cases, the two-dimensional Euclidean space is measured by the linear meter ${ }^{53}$ and we apply for it the mathematical rules. This does not appear as true from the point of view of the Philosophy of Science ${ }^{54}$ as it regards two ontological and joint formulation terms of "Transport" and "Logistics", such as "Time" and "Space", which belong to the field of empathetic anthropological perception rather than the linear measurement.

The terminological confusion between Science and Technology has often made to look like as "Science" what, in reality, is and it remains as Technology ${ }^{55}$, which was born

For logistics system one means all supply, production, storage, transport and distribution of goods centers and their interactions (trade flows). Currently it tends to distinguish:

- The logistics of supply, which takes place "upstream" of the production facilities, and consists in optimizing the management of supplies of raw materials, parts and shapes, depending on the production plan of the company.

- The internal logistics, which takes place within the production facilities. It consists in the optimization of production processes: receiving, storage and handling of materials and processing; collection of materials from stock to feed the production lines, handling of semi-finished; packaging of finished products.

- The logistics of distribution, which takes place "downstream" of the production facilities and "upstream" of the market: it consists in optimizing the supply of retail outlets or direct delivery to end customer (M. Ferrarese, ibidem, $10^{\text {th }}$ lesson).

${ }_{51}$ In mathematics, reductionism can be interpreted as the philosophy that all mathematics can (or ought to) be built on a common foundation, which is usually axiomatic set theory. Ernst Zermelo was one of the major advocates of such a view; he also developed much of axiomatic set theory. It has been argued that the generally accepted method of justifying mathematical axioms by their usefulness in common practice can potentially undermine Zermelo's reductionist program (http://en.wikipedia.org/wiki/Reductionism )$_{52}$

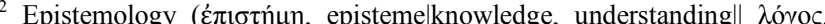
logos(study of) is concering the nature and scope of knowledge and it is also referred to as "Theory of Knowledge"... American philosopher Robert Nozick (November 16, 1938 - January 23, 2002 ) has offered his "tracking theory" of knowledge as the Four Conditions (with third and fourth conditions as "counterfactuals") for $S$ 's knowing that $P$ were (i.e. $S$ knows that $P$ if and only if):

- $\quad P$ is true

- $\quad$ S believes that $\mathrm{P}$

- If it were the case that (not-P), $\mathrm{S}$ would not believe that $\mathrm{P}$

- If it were the case that $\mathrm{P}, \mathrm{S}$ would believe that $\mathrm{P}$.

${ }^{53}$ Now, as the length of the path travelled by light in vacuum during a time interval of $1 / 299,792,458$ of a second.

${ }^{54}$ Philosophy of Science is a branch of philosophy concerned with the foundations, methods, and implications of science. The central questions concern what counts as science, the reliability of scientific theories, and the purpose of science (http://en.wikipedia.org/wiki/Philosophy of science).

The distinction between science, engineering and technology is not always clear. Science is the reasoned investigation or study of phenomena, aimed at discovering enduring principles among elements of the phenomenal world by employing formal techniques such as the scientific from empirical experience and generalized through the application of mathematical models: eg. the flow transportation models of Greenshield and Greenberg, the parabolic model, the logarithmic model, the flows uninterrupted model, the neural networks, and so on. All these models seek a mathematic accommodation of empirical observations, which was born "tunc pro nunc" (then instead of now) with the ambition to hold for ever, to be "evergreen". Nothing could be more wrong from the point of view of the Philosophy of Science and, therefore, of the Ontological Science.

With our work we tried to ennoble "Technology", that is defined as "Applied Science" in the United States, making it rise to the rank of "Science of Transport and Logistics", by the epistemological formulation, trying to give a scientific body to the whole matter and developing a doctrinal system ${ }^{56}$, where, since now, there's been existing only some tracks and bit (epistemological) insights.

Think of Transport and Logistics as "Technology" is only as reductive: the debate on Time and Space in Transport and Logistics is well away from the pure field measurements. It belongs to the psychological and anthropological spheres and it should be treated as such. Where is Man, over there it is Thought and the definition of a Time and a Space: the Science of Transport and Logistics begins where we study Ontological Space and Time for Man.

\section{REFERENCES}

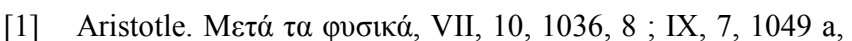
27.

[2] Baudrillard, J. (1976). La società dei consumi. Bologna: Il Mulino.

[3] Ellington, J.W. (1785). Grounding for the Metaphysics of Morals in Kant. 3rd ed.

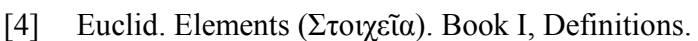

[5] Ferrarese, M. (2010). Course of Transport and Mobility Economics - CPS. Verona: Transmit, 2nd class).

[6] Fraenkel, A., Bar-Hille, Y., Levy, A. (1973). Foundations of Set Theory. Fraenkel's final word on ZF and ZFC. North Holland.

method. Technologies are not usually exclusively products of science, because they have to satisfy requirements such as utility, usability and safety...Technology is often a consequence of science and engineering although technology as a human activity precedes the two fields.... The exact relations between science and technology in particular have been debated by scientists, historians, and policymakers in the late 20th century, in part because the debate can inform the funding of basic and applied science. ... In the United States it was widely considered that technology was simply "Applied Science" and that to fund basic science was to reap technological results in due time...(http://en.wikipedia.org/wiki/Technolog y).

56 As like other "Sciences" of recent formulation, such as, for example, Business Administration, this matter has risen to Science through the contribution of the epistemological studies of Gino Zappa (Zappa, 1926, 1957). 
[7] Fromm, E. (1980). Avere o essere? Segrate: Mondadori.

[8] Horace. Odes 1, 11, 8.

[9] ISTAT, The Italian Accounts. (2001). Bologna: Il Mulino.

[10] Kant. Transcendental Analytic, 24.

[11] Kobayashi, S., Nomizu, K. (1996). Foundations of Differential Geometry. Wiley-Interscience, Vol. 1.

[12] Longo, G. (2008). Some Aspects of Time in Physics and Philosophy, or some thoughts of a physicist on a problem as old as time. Paper.

[13] Minkowski, H. (1908). Speech of 21 September 1908 at Assembly of Nature Scientists and Germans Doctors.

[14] Nietzsche, F. (1988). Ditirambi di Dioniso. Milan: Adelphi, 133.

[15] Paolucci, G. (1989). "Una figura della temporalità moderna: la scarsità del tempo", in: Belloni M.C., Rampazi M. Tempo, spazio, attore sociale. Tredici saggi per discutere. Milan: Franco Angeli,

[16] Peri, P. (2001). Progetto di ricerca su esigenze e aspettative degli utenti giornalieri delle ferrovie rispetto ai servizi loro offerti e ai materiali rotabili utilizzati. Dipartimento di Sociologia e Ricerca Sociale dell'Universita' degli Studi di Trento. Transcendental Analytic, 24.

[17] Stock, J., Watson, M. (2005). Introduzione all'econometria. Milano: Pearson Education, pp. 100.

[18] Thoreau, H.D. (1854). A Week on the Concord and Merrimack Rivers / Walden / The Maine Woods. Boston: Ticknor and Fields.

[19] Zappa, G. Tendenze nuove negli studi di ragioneria, Discorso inaugurale dell'Anno Accademico1926-27 nel Regio Istituto Superiore di Scienze Economiche e Commerciali di Venezia,Venice, doctoral paper, 1926.

[20] Zappa, G. Le produzioni nell'economia delle imprese , Giuffrè, Milan, 1957, Tomo I.

[21] http://www.istat.it/it/archivio/spostamenti

[22] http://it.wikipedia.org/wiki/Deflusso_veicolare

[23] http://en.wikipedia.org/wiki/Philosophy_of_science

[24] http://en.wikipedia.org/wiki/Technology 